\title{
Studying the Safety Impact of Autonomous Vehicles Using Simulation-Based Surrogate Safety Measures
}

\author{
Mark Mario Morando, ${ }^{1}$ Qingyun Tian, ${ }^{2}$ Long T. Truong ${ }^{(D)}{ }^{1}$ and Hai L. Vu ${ }^{1}$ \\ ${ }^{1}$ Monash Institute of Transport Studies, Department of Civil Engineering, Monash University, Melbourne, VIC, Australia \\ ${ }^{2}$ School of Civil and Environmental Engineering, Nanyang Technological University, Singapore \\ Correspondence should be addressed to Long T. Truong; long.truong@monash.edu
}

Received 30 November 2017; Revised 11 February 2018; Accepted 20 February 2018; Published 22 April 2018

Academic Editor: Abdelaziz Bensrhair

Copyright (c) 2018 Mark Mario Morando et al. This is an open access article distributed under the Creative Commons Attribution License, which permits unrestricted use, distribution, and reproduction in any medium, provided the original work is properly cited.

\begin{abstract}
Autonomous vehicle (AV) technology has advanced rapidly in recent years with some automated features already available in vehicles on the market. AVs are expected to reduce traffic crashes as the majority of crashes are related to driver errors, fatigue, alcohol, or drugs. However, very little research has been conducted to estimate the safety impact of AVs. This paper aims to investigate the safety impacts of AVs using a simulation-based surrogate safety measure approach. To this end, safety impacts are explored through the number of conflicts extracted from the VISSIM traffic microsimulator using the Surrogate Safety Assessment Model (SSAM). Behaviours of human-driven vehicles (HVs) and AVs (level 4 automation) are modelled within the VISSIM's carfollowing model. The safety investigation is conducted for two case studies, that is, a signalised intersection and a roundabout, under various $\mathrm{AV}$ penetration rates. Results suggest that AVs improve safety significantly with high penetration rates, even when they travel with shorter headways to improve road capacity and reduce delay. For the signalised intersection, AVs reduce the number of conflicts by $20 \%$ to $65 \%$ with the AV penetration rates of between $50 \%$ and $100 \%$ (statistically significant at $p<0.05$ ). For the roundabout, the number of conflicts is reduced by $29 \%$ to $64 \%$ with the $100 \%$ AV penetration rate (statistically significant at $p<0.05)$.
\end{abstract}

\section{Introduction}

Autonomous vehicle (AV) technology has advanced significantly in recent years. Automakers have already provided vehicles with some automated features (e.g., self-parking) and crash avoidance features such as automated braking, forward collision warning, lane departure warning, and blind spot monitoring $[1,2]$. AV testing and piloting have begun in various countries. By 2014, AV testing on roadways has been legalised in four states in the US. In Australia, AV testing has been first introduced in South Australia's roadways in 2016 [3]. The market penetration rate of AVs is estimated to be between $24 \%$ and $87 \%$ by $2045[4,5]$.

AVs have the potential to significantly improve road safety as the majority of crashes are related to driver errors, fatigue, alcohol, or drugs [6-8]. It is also expected that AVs can travel with shorter headways due to improved safety, leading to increased road and intersection capacities $[9,10]$.
AVs would also provide improved mobility to the disabled, those who are too young to drive, and older people [11]. Other potential benefits of AVs include enhanced productive use of travel time, fewer emissions, better fuel efficiency, and reduced parking costs $[12,13]$.

Implementing AVs within the road network has the potential to significantly reduce the number of crashes caused by the drivers through the gradual removal of human control [12]. Already, many vehicle manufacturers are increasing the implementation of features such as adaptive cruise control and parking assistance that enables the vehicle to park itself with minimal human intervention [14]. Many of these driver assistance features are partially automated, meaning that driver intervention is still required. Although the implementation of automated features has increased in recent years, fully AVs are yet to be legally deployed on a large scale within the road network globally. Safety benefits of fully AVs would not be maximised without a high penetration rate of AVs [12]. 
It should be noted that even as continuous developments are made to AV software by frontrunners such as Google and Tesla, questions arise with regard to their ability to produce a fleet of fully automated driving systems that are safe enough to negate the need for human intervention [15]. Recent research suggested that current levels of technology from frontrunners like Google and Mercedes-Benz still experience malfunctions that require the need for human intervention [16]. Further issues arise with regard to the amount of miles the cars will need to be tested for in order to obtain statistically relevant data to demonstrate the vehicles' safety. Before these vehicles are able to be deployed within road networks, it is expected that statistically relevant data must be collected to certain standards. This may be a major setback as the amount of testing required to validate a particular failure rate could be of great magnitude [15]. In addition, the safety implications in terms of network security will need to be addressed as cyberattacks on connected AVs are a major safety threat [15]. Due to the connected nature of such advanced vehicle technology, an area that needs to be addressed is the potential for such attacks causing further safety risks, counteracting the theorised safety improvement from the elimination of human control.

Several studies have attempted to examine safety benefits of AVs using different approaches. Fagnant and Kockelman [14] assumed near elimination of human errors, which is related to main factors of over $90 \%$ of crashes in the US, from AV technology. Rau et al. [17] developed a method to identify crashes, which could be addressed by AV technology, by mapping automated vehicle functions to five layers of crash information (location, precrash scenario, driving conditions, travel speed, and driver condition). Real-world data from AV testing in California has increasingly been utilised in recent research [16, 18-20]. For example, Schoettle and Sivak [18] found that AVs were not at fault in any crashes and the overall injury severity was lower for crashes involving AVs than for crashes involving human-driven vehicles (HVs).

Potential safety benefits of different connected vehicle technology and control have been evaluated in previous research. Tian et al. [21] showed that platoon-based driving can significantly reduce the chain collisions and alleviate their severity. Optimising vehicle trajectories via adjusting the platoon level reaction time and speed of the lead vehicle was shown to provide both efficiency and safety benefits [22]. It is worth noting that these studies only considered single lane traffic. Zhou et al. [23] investigated the impacts of connected AVs on the performance of freeway merging. Safety impacts were also considered, but indirectly through impacts on speed dispersion. As previous research tends to focus on freeways, there is a lack of investigations on other network settings, such as signalised intersections and roundabouts.

Using traffic microsimulation models of various network settings, Kockelman et al. [24] found that in general AVs reduce the number of potential conflicts based on surrogate safety measures and thus improve safety. In their study, a car-following model based on the Wiedemann 74 and 99 models was adopted for HVs. This model was then modified to model behaviours of AVs. For example, as AV behaviours are expected to be less stochastic, the variance of the driver random terms was set to zero. Other parameters, such as minimum acceptable gap for merging or turning, sight distance, and lane change preferences, were modified to make the AV behaviours more conservative as automakers would be very unlikely to make AVs aggressive due to their potential liability. In other words, AVs were modelled to be more cautious than human drivers and therefore had fewer potential conflicts. However, there is another possible scenario, particularly in the long run, where AV behaviours could be less conservative due to shorter headways and more aggressive acceleration as AVs are anticipated to increase road and intersection capacities. Thus, it is also important to investigate the safety impacts of AVs with such behaviours.

This paper aims to investigate safety impacts of AVs using simulation-based surrogate safety measures where AVs are modelled with anticipated behaviours, such as shorter headways. Safety performance of AVs is considered with varying penetration rates in two case studies, that is, a signalised intersection and a roundabout. The delay reduction at increasing penetration rates will also be considered.

The remainder of this paper is organized as follows. In the next section, the methodology will be outlined, followed by key results and discussion. The paper is concluded with a summary of key findings and directions for future research.

\section{Methodology}

To understand the safety implications of AVs, VISSIM [25] was used as the traffic microsimulation platform while Surrogate Safety Assessment Model (SSAM) [26] was employed to extract the number of potential conflicts based on surrogate safety measures from simulated data. The following subsections present the simulation platform, surrogate safety measure extraction, and then the case studies.

2.1. Simulation Platform. Behaviours of human-driven vehicles (HVs) were modelled using VISSIM's Wiedemann 99 car-following model with default parameters. This provides a reasonable base model for human drivers. This paper assumes that AVs are fully automated with level 4 automation [27]. Previous studies have shown that parameters of the VISSIM car-following model can be modified to model behaviours of AVs [2, 28]. Two sets of AV parameters adopted from Atkins [28] and PTV [29] were considered in this study. Table 1 presents a set of $\mathrm{HV}$ parameters and two sets of AV parameters.

Parameters for AVs reflect more assertive behaviours, such as shorter standstill distance (CC0) and shorter safety distance (lower headway CC1 and following variation CC2). Thus, shorter gaps are expected for AVs (Figure 1). Smaller values of the negative following threshold (CC4) and positive following threshold (CC5) reflect a more sensitive reaction of AVs to the acceleration or deceleration of the preceding vehicle. As AVs can strictly follow the desired speed without oscillation, CC6 is set as zero. According to Atkins [28], AVs can also have more aggressive acceleration (higher CC7 and CC8) and a higher number of observed vehicles due to connected vehicle technology. Although the exact behaviours 
TABLE 1: Parameters for HV and AV behaviours.

\begin{tabular}{|c|c|c|c|c|}
\hline Parameter & Description & $\mathrm{HV}$ & $\mathrm{AV}-1$ & $\mathrm{AV}-2$ \\
\hline CC0 standstill distance $(\mathrm{m})$ & The desired distance between stopped vehicles & 1.5 & 0.5 & 0.75 \\
\hline CC1 headway time (s) & The gap in seconds that a vehicle keeps & 0.9 & 0.5 & 0.45 \\
\hline CC2 following variation $(\mathrm{m})$ & $\begin{array}{l}\text { The distance in addition to the allowed safety distance that is } \\
\text { permissible before the vehicle-drive unit moves closer to the } \\
\text { proceeding vehicle }\end{array}$ & 4 & 0 & 2 \\
\hline CC4 negative following threshold & Control speed differences during car following & -0.35 & 0 & -0.1 \\
\hline CC5 positive following threshold & Control speed differences during car following & 0.35 & 0 & 0.1 \\
\hline $\begin{array}{l}\text { CC6 speed dependency of } \\
\text { oscillation }\end{array}$ & $\begin{array}{l}\text { Influence of distance on speed oscillation (the variation of speed } \\
\text { around the desired speed) }\end{array}$ & 11.44 & 0 & 0 \\
\hline $\operatorname{CC7}\left(\mathrm{m} / \mathrm{s}^{2}\right)$ & Influence of vehicle acceleration during car-following oscillation & 0.25 & 0.45 & 0.25 \\
\hline $\mathrm{CC} 8\left(\mathrm{~m} / \mathrm{s}^{2}\right)$ & Desired acceleration when starting from standstill & 3.5 & 3.9 & 3.5 \\
\hline Look-ahead distance & Number of observed vehicles the model will look ahead at & 2 & 10 & 2 \\
\hline
\end{tabular}

Note. HVs are VISSIM default parameters; AV-1 are adopted from Atkins [28]; AV-2 are adopted from PTV [29].
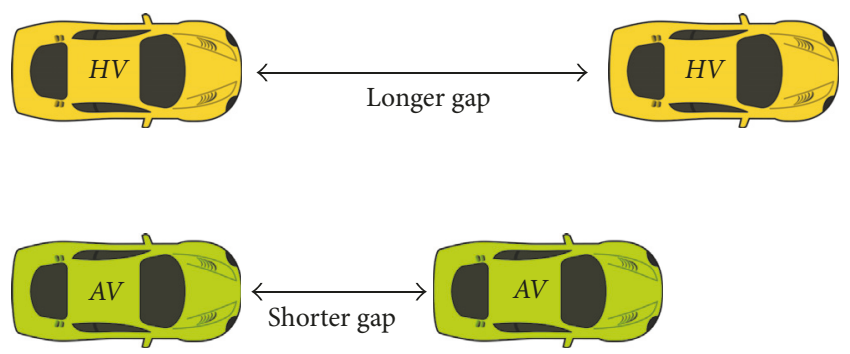

FIGURE 1: Shorter gaps between AVs.

of AVs are largely unknown at this stage, the modification of these parameters should be able to reflect the anticipated AV behaviours.

2.2. Surrogate Safety Measures. Surrogate safety measures are tools that can be used to assess potential conflicts of a road network. Surrogate safety measures, such as time to collision (TTC), postencroachment time (PET), deceleration rate (DR), gap time (GT), and proportion of stopping distance (PSD), are useful measurements of the safety implications [30-33]. TTC, defined as the expected time for two vehicles to collide if they remain on the same path and at the same speed, is a widely used surrogate safety measure. Figure 2 illustrates situations for TTC calculations [34]. The following equation can be used to calculate TTC:

TTC

$$
= \begin{cases}\frac{d_{2}}{v_{2}} & \text { if } \frac{d_{1}}{v_{1}}<\frac{d_{2}}{v_{2}}<\frac{d_{1}+l_{1}+w_{2}}{v_{1}} \text { (side) } \\ \frac{d_{1}}{v_{1}} & \text { if } \frac{d_{2}}{v_{2}}<\frac{d_{1}}{v_{1}}<\frac{d_{2}+l_{2}+w_{1}}{v_{2}} \text { (side) } \\ \frac{X_{1}-X_{2}-l_{1}}{v_{2}-v_{1}} & \text { if } v_{2}>v_{1} \text { (rear end) } \\ \frac{X_{1}-X_{2}}{v_{1}+v_{2}} & \text { (head on), }\end{cases}
$$

where $v_{1}$ and $v_{2}$ are vehicle speeds, $l_{1}$ and $l_{2}$ are vehicle lengths, $w_{1}$ and $w_{2}$ are vehicle widths, $X_{1}$ and $X_{2}$ are vehicle positions, and $d_{1}$ and $d_{2}$ are distances to conflict areas.

PET, defined as the time difference between when the leading vehicle occupies a location and when the trailing vehicle arrives at this location, is usually used to identify conflicts in combination with TTC [35]. Figure 3 illustrates situations for PET calculations [34].

It is generally considered that a TTC, equal to or less than 1.5 seconds, would result in an unsafe situation [26, 36, 37]. This threshold of 1.5 seconds is therefore applied for potential conflicts involving HVs (HV-HV and HV-AV). It can be argued that the TTC threshold for conflicts between AVs would be lower due to their ability to react to situations a lot faster that their human counterparts, particularly with connected vehicle technology. Therefore, a sensitivity analysis for the TTC threshold of AV-AV conflicts was conducted with two values ( 1 second and 0.75 seconds). A PET threshold of 5 seconds is used as the default value in SSAM [26]. Overall, a potential conflict is identified when the calculated TTC and PET are equal to or smaller than their respective threshold.

SSAM was developed for analysing simulation-based surrogate safety measures [26]. It works through the analysis of trajectory files (.trj) obtained from various traffic microsimulation programs, such as Aimsun, Paramics, and VISSIM. In this study, the trajectory files were obtained from VISSIM, which were then imported into the SSAM to identify the number of potential conflicts. Although SSAM does not have a built-in function to identify conflicts by vehicle types, it does provide the ID of vehicles involved in a conflict. Vehicle IDs and corresponding vehicle types can be extracted from VISSIM. Thus, it was possible to identify the number of potential conflicts by vehicle types (HV-HV, HV-AV, and AV$\mathrm{AV})$.

2.3. Case Studies. Using the aforementioned approach, safety impacts of autonomous vehicles were investigated in two case studies, that is, a signalised intersection and a roundabout. Details of each case study are presented in the following subsections. 

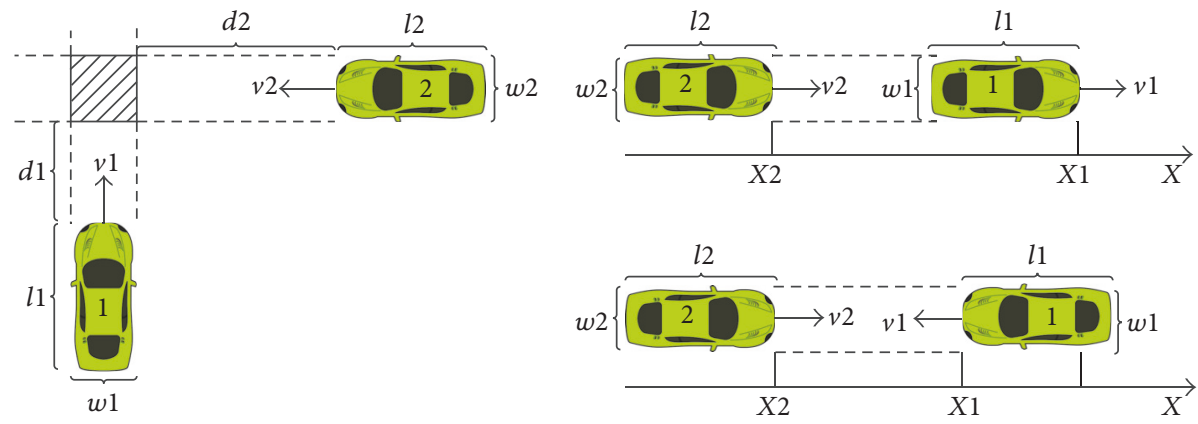

FIgUre 2: Time to collision (TTC).

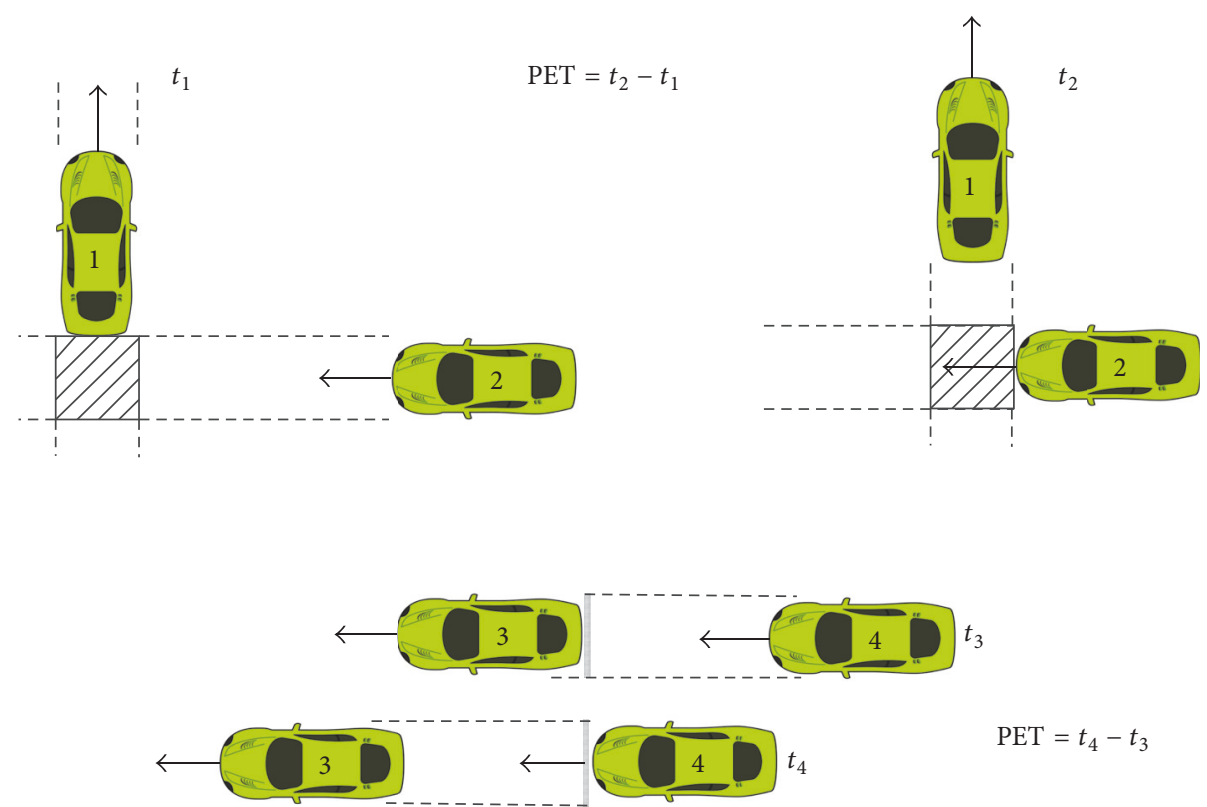

Figure 3: Postencroachment time (PET).

2.3.1. Signalised Intersection. The intersection of Ferntree Gully Road and Blackburn Road in Melbourne, Australia, was chosen as the first case study. Both roads are 3-lane arterial roads with several bus routes. The intersection also has a right turning lane on all four approaches and a left turning slip lane on three approaches. To ensure the model replicates the intersection as closely as possible, SCATS data (traffic volume and traffic signal phasing) for the peak period between 8 and 9 a.m. was obtained from VicRoads to calibrate the model. Traffic volumes per intersection approach range from 760 to $2260 \mathrm{veh} / \mathrm{h}$. The traffic composition was $95 \%$ cars and $5 \%$ trucks. The bus routes with far-side and midblock bus stops were also modelled with scheduled arrival times. The intersection layout is illustrated in Figure 4.

The variance in desired speed distributions is another key element that varies from HVs and AVs within the network. It can be assumed that the variation in the desired speed distribution of a human driver would be much larger than that of an AV due to the nature of AVs having much more precise throttle control. Based on field conditions, the desired speed distribution for HVs ranges between $65 \mathrm{~km} / \mathrm{h}$ and

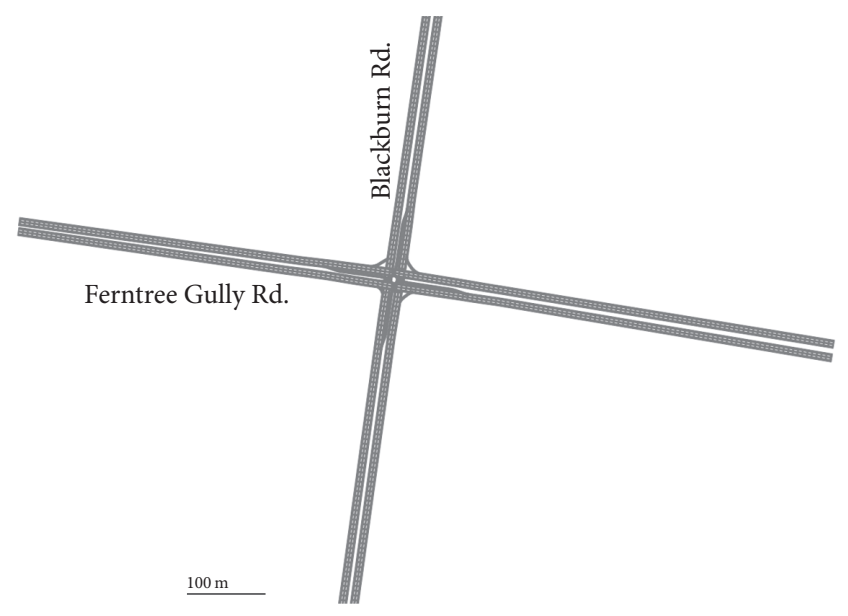

FIGURE 4: Signalised intersection case study.

$75 \mathrm{~km} / \mathrm{h}$. AVs' desired speed distribution has the same mean, but a narrower range between $69 \mathrm{~km} / \mathrm{h}$ and $71 \mathrm{~km} / \mathrm{h}$. 
TABLE 2: Conflicts by types and AV penetration rates for the signalised intersection (AV-1 parameters and $0.75 \mathrm{~s}$ TTC threshold for AV-AV conflicts).

\begin{tabular}{|c|c|c|c|c|}
\hline \multirow{2}{*}{ Penetration rate } & \multicolumn{3}{|c|}{ Conflict type } & \multirow{2}{*}{ Total } \\
\hline & AV-AV & AV-HV & HV-HV & \\
\hline $0 \%$ (base case) & & & 863.3 & 863.3 \\
\hline $25 \%$ & 23.9 & 326.7 & 365.7 & 716.2 \\
\hline $50 \%$ & 85.8 & 389.1 & 148.2 & 623.1 \\
\hline $75 \%$ & 182.1 & 286.4 & 41.7 & 510.1 \\
\hline $100 \%$ & 411.9 & & & 411.9 \\
\hline
\end{tabular}

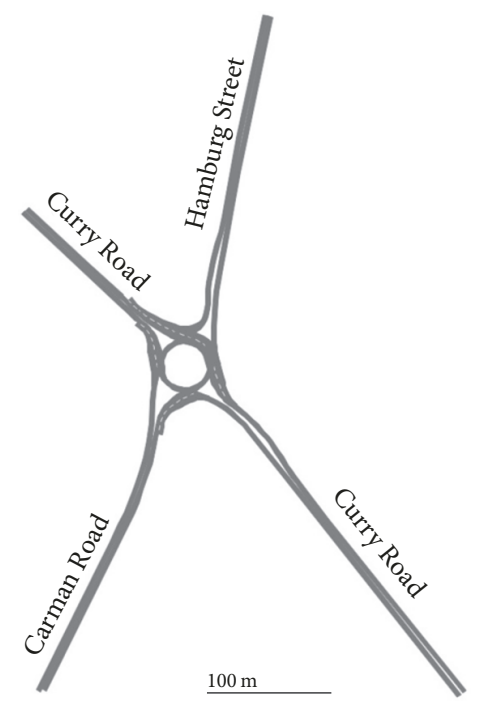

FIgURE 5: Roundabout case study.

2.3.2. Roundabout. The second case study was based on a roundabout model that was provided with SSAM. This is a four-legged roundabout in Schenectady, New York. Traffic volumes per lane on each approach range from about 490 to $1050 \mathrm{veh} / \mathrm{h}$. Traffic composition was $96 \%$ cars and $4 \%$ trucks. The desired speed distribution for HVs ranges between $48 \mathrm{~km} / \mathrm{h}$ and $58 \mathrm{~km} / \mathrm{h}$. Like the first case study, AVs' desired speed distribution has a narrower range between $52 \mathrm{~km} / \mathrm{h}$ and $54 \mathrm{~km} / \mathrm{h}$. It is noted that desired speeds within the roundabout were modelled to be lower (between 20 and $25 \mathrm{~km} / \mathrm{h}$ ), reflecting reduced speed behaviours. Figure 5 depicts the layout of the roundabout.

2.3.3. Scenarios. Varying penetration rates of $\mathrm{AVs}$, including $0 \%$ (base case with $100 \% \mathrm{HVs}$ ), $25 \%, 50 \%, 75 \%$, and $100 \%$, were analysed for each case study. For the signalised intersection case study, all buses were modelled with AV behaviours in the $100 \% \mathrm{AV}$ scenarios, but with HV behaviours in other scenarios. In total, there were 18 scenarios. For each scenario, the simulation time was set to 1 hour, excluding a warmup time of 10 mins. All scenarios were modelled with 10 simulation time steps per second. This maximum simulation time step was used to better accommodate AVs' shorter headways. Due to the stochastic nature of microsimulation, 20 runs with different seed numbers were performed for each scenario to obtain reliable outputs [38].

\section{Results and Discussion}

\subsection{AV-1 Parameters}

3.1.1. Signalised Intersection. Results for the first AV parameter set (AV-1) with the TTC threshold for AV-AV conflicts of 0.75 seconds are presented in Table 2. As expected, the number of conflicts between AVs and AVs increases and the number of conflicts between HVs and HVs decreases with increasing $\mathrm{AV}$ penetration rates. At the penetration rate of $50 \%$, the number of conflicts between AVs and HVs is maximum. Conflicts between AVs and HVs appear more frequently with the $25 \% \mathrm{AV}$ penetration rate than with the $75 \% \mathrm{AV}$ penetration rate. The reason may be that, with the $75 \% \mathrm{AV}$ penetration rate, an $\mathrm{AV}$ is more likely to have a conflict with another AV due to shorter headways.

Figures 6(a) and 6(b) depict the total number of conflicts and associated 95\% confidence intervals (CIs) by varying AV penetration rates for both the 0.75 -second and 1-second TTC thresholds. It is evident that the number of conflicts in all AV scenarios is lower compared to the base case. Safety benefits from $\mathrm{AV}$ s range between $15 \%$ and $52 \%$, depending on penetration rates and TTC threshold for AV-AV conflicts. Moreover, the number of conflicts with the $50 \%, 75 \%$, and $100 \% \mathrm{AV}$ penetration rates is significantly lower compared to the base case, at $p<0.05$. With regard to the TTC threshold of 1 second for AV-AV conflicts, the number of conflicts with the $75 \%$ penetration rate is slightly lower than that with the $50 \%$ and $100 \%$ penetration rates (Figure 6(a)). However, these differences are not statistically significant. Compared to the base case, the number of conflicts is reduced by around $20 \%$ with the $100 \%$ AV penetration rate. These results suggest that safety benefits of AVs might not be significant until a high penetration rate is achieved. However, results with the TTC threshold of 0.75 seconds for AV-AV conflicts show a consistent decline in the number of vehicle conflicts as the penetration rate is increased to $100 \%$ (Figure 6(b)). At a penetration rate of $50 \%$ and above, the decline is statistically significant at $p<0.05$. This is further evidence that higher penetration rates of AVs are likely to provide safety benefits.

Overall, while the results demonstrate that the TTC threshold for AV-AV conflicts is a major factor in identifying potential conflicts, they consistently show the safety benefits of AVs, particularly with high AV penetration rates.

3.1.2. Roundabout. Table 3 summarises the average number of conflicts by different types and $\mathrm{AV}$ penetration rates for 


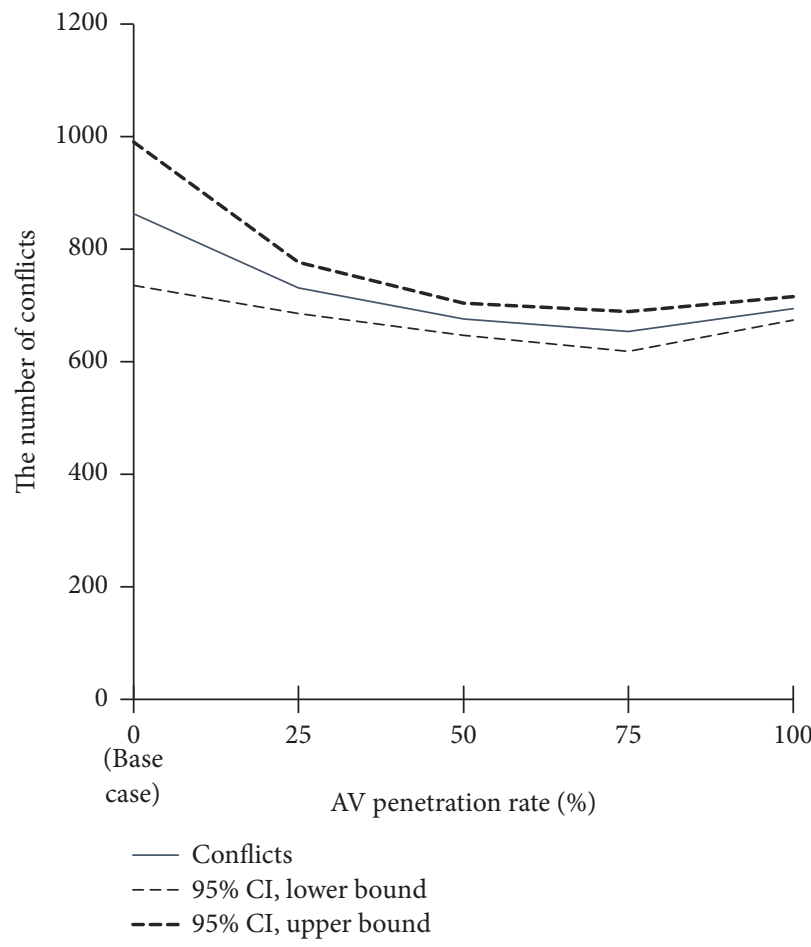

(a) $1 \mathrm{~s}$ TTC threshold for AV-AV conflicts

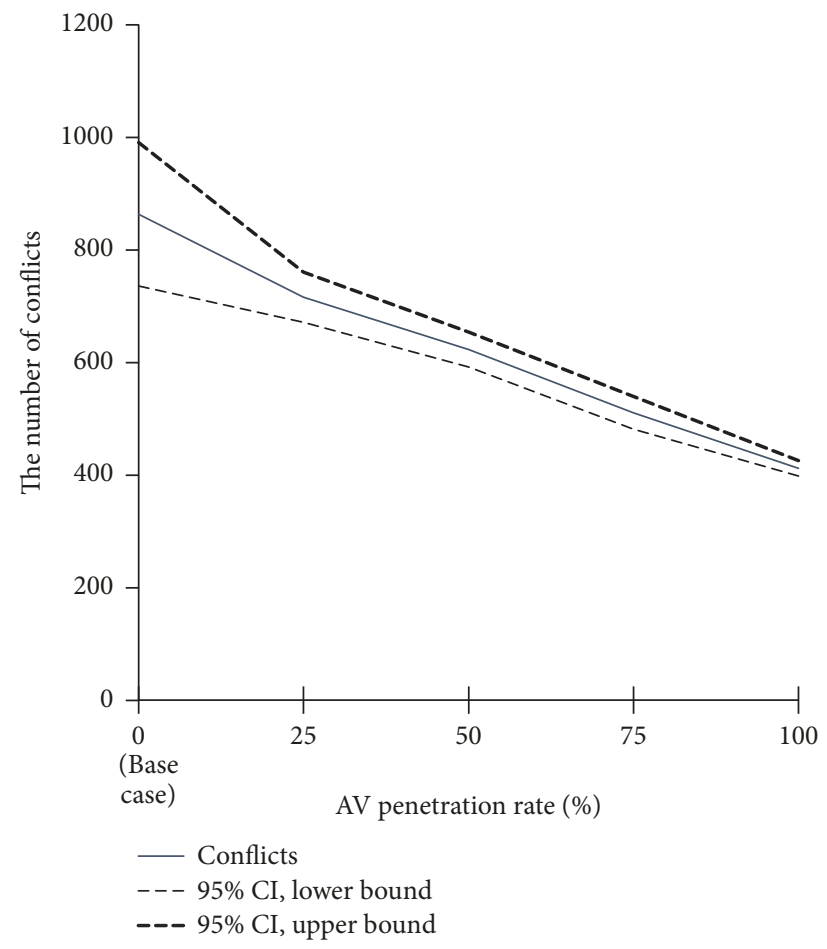

(b) $0.75 \mathrm{~s}$ TTC threshold for AV-AV conflicts

FIGURE 6: Total number of conflicts by AV penetration rate for the signalised intersection (AV-1 parameters).

TABLE 3: Conflicts by types and AV penetration rates for the roundabout (AV-1 parameters and $0.75 \mathrm{~s}$ TTC threshold for AV-AV conflicts).

\begin{tabular}{|c|c|c|c|c|}
\hline \multirow{2}{*}{ Penetration rate } & \multicolumn{3}{|c|}{ Conflict type } & \multirow{2}{*}{ Total } \\
\hline & AV-AV & AV-HV & HV-HV & \\
\hline $0 \%$ (base case) & & & 449.1 & 449.1 \\
\hline $25 \%$ & 14.8 & 270.1 & 165.0 & 449.9 \\
\hline $50 \%$ & 48.7 & 275.9 & 83.0 & 407.6 \\
\hline $75 \%$ & 108.5 & 204.7 & 18.7 & 331.9 \\
\hline $100 \%$ & 172.9 & & & 172.9 \\
\hline
\end{tabular}

the roundabout case study (TTC threshold of 0.75 seconds for $\mathrm{AV}-\mathrm{AV}$ conflicts). As the penetration rate increases, the number of conflicts between AVs increases whereas the number of conflicts between HVs decreases. Similar to results of the signalised intersection, the number of conflicts between $\mathrm{AV}$ and $\mathrm{HV}$ is highest with the $50 \%$ penetration rate. These results are expected. The total number of conflicts by varying AV penetration rates is further depicted in Figure 7.

Figures 7(a) and 7(b) with different TTC thresholds show that the number of conflicts increases between the base case and $25 \% \mathrm{AV}$ penetration rate but decreases steadily between $25 \%$ and $75 \% \mathrm{AV}$ penetration rates. In particular, the number of conflicts with $100 \% \mathrm{AV}$ penetration rate is significantly lower at $p<0.05$, compared to other AV penetration rates. Considering the TTC threshold of 1 second for AVAV conflicts, AVs provide safety benefits by $6 \%$ and $29 \%$ with the penetration rate of $75 \%$ and $100 \%$, respectively, compared to the base case (Figure $7(\mathrm{a})$ ). With regard to the AV-AV TTC threshold of 0.75 seconds (Figure 7(b)), the results show increased safety benefits of between $9 \%$ and $62 \%$ for penetration rates of $50 \%$ and $100 \%$, respectively. These results also confirm that a high $\mathrm{AV}$ penetration rate might be required to deliver AVs' anticipated safety benefits.

Different patterns in conflicts by penetration rates between the two case studies suggest that safety impacts of AVs might vary with different networks. Thus, it is important for future research to conduct further investigations with various network settings and traffic conditions. With the $100 \%$ AV penetration rate, safety benefits are slightly higher with the roundabout than with the signalised intersection (62\% versus $52 \%$ ). This suggests that roundabouts might be preferred in terms of safety when all vehicles are AVs.

3.1.3. Delay Results. Figure 8 shows the delay results at varying penetration rates. It is evident that as the penetration rate of AVs is increased, there is a clear decrease in delay for both the roundabout and the signalised intersection. Furthermore, the decreases in delay with increasing penetration rates are statistically significant at $p<0.05$. This is expected given that AVs were modelled to travel with shorter headways. 


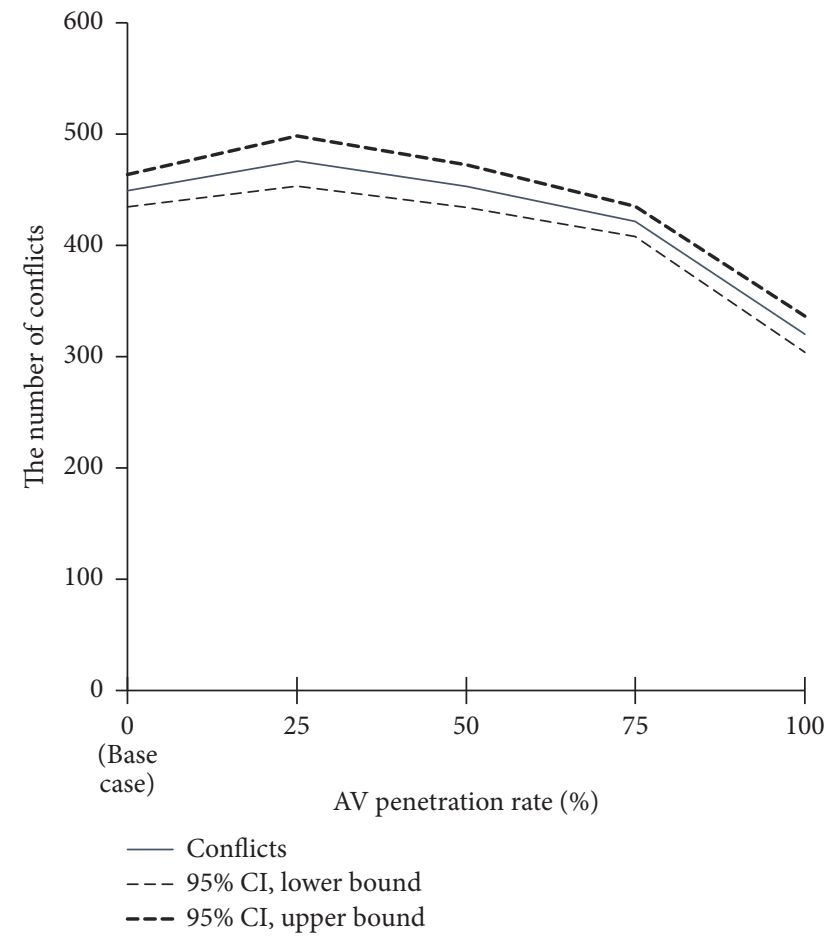

(a) $1 \mathrm{~s}$ TTC threshold for AV-AV conflicts

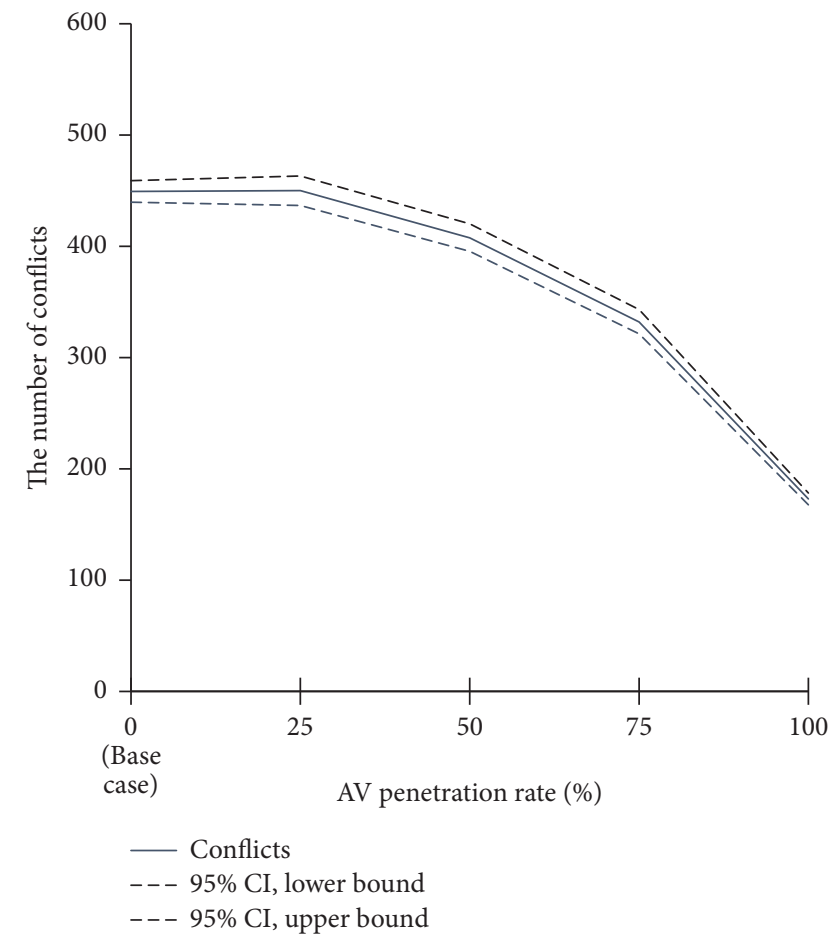

(b) $0.75 \mathrm{~s}$ TTC threshold for AV-AV conflicts

FiguRE 7: Total number of conflicts by AV penetration rate for the roundabout (AV-1 parameters).

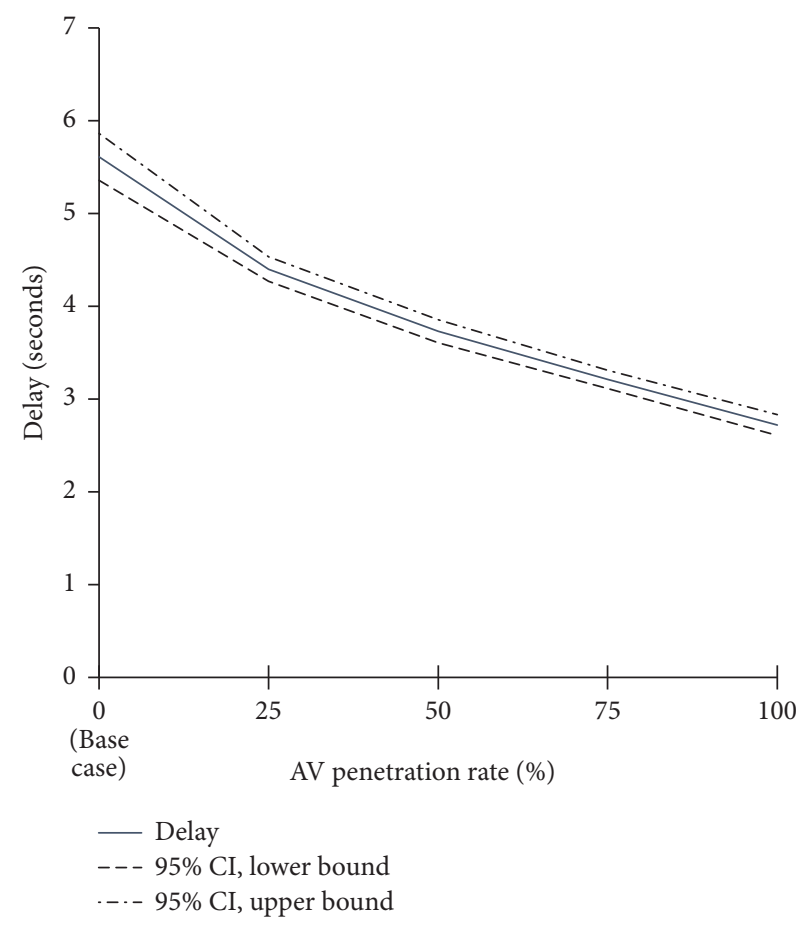

(a) Roundabout delay

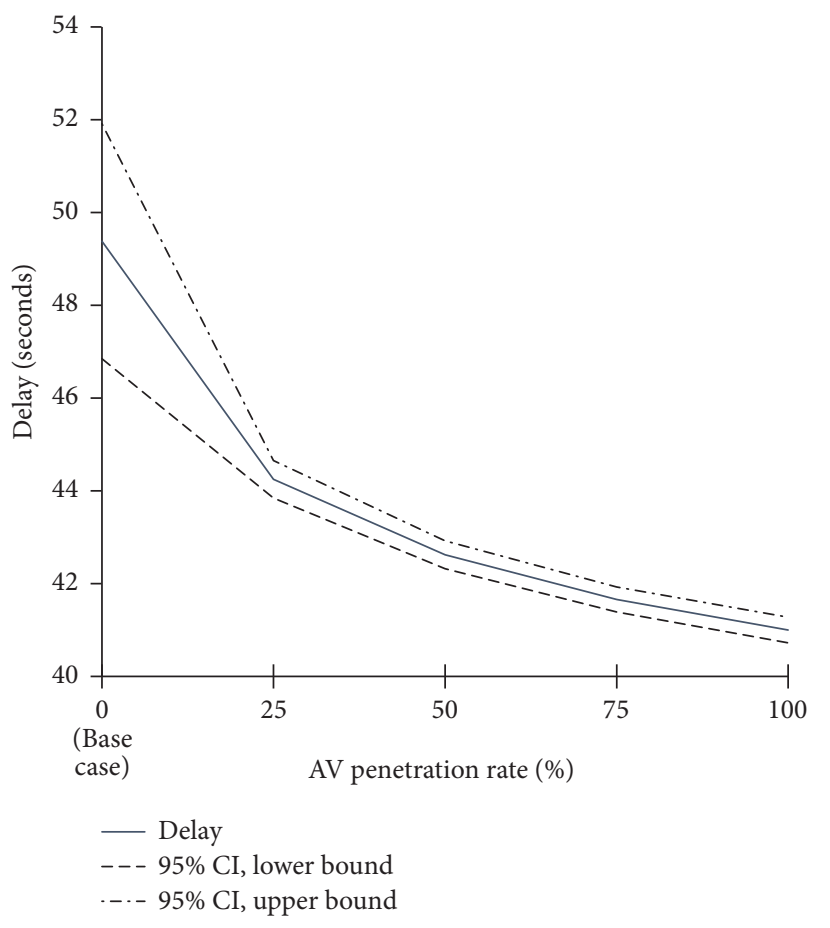

(b) Signalised intersection delay

FIgURE 8: Average roundabout and intersection delay by penetration rate. 


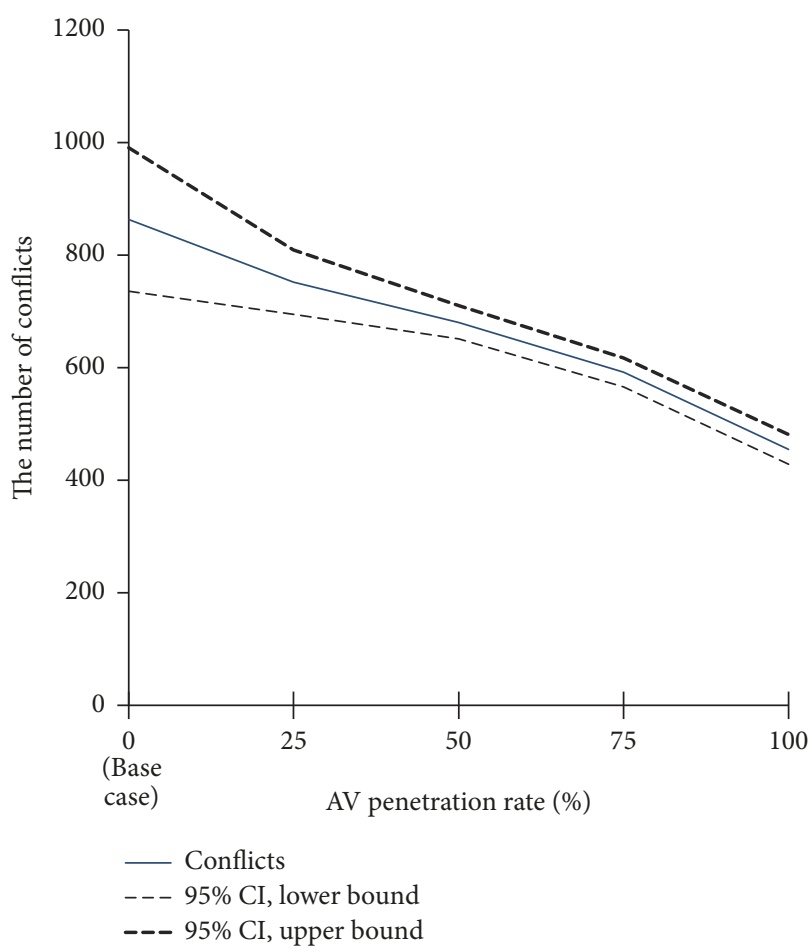

(a) $1 \mathrm{~s}$ TTC threshold for AV-AV conflicts

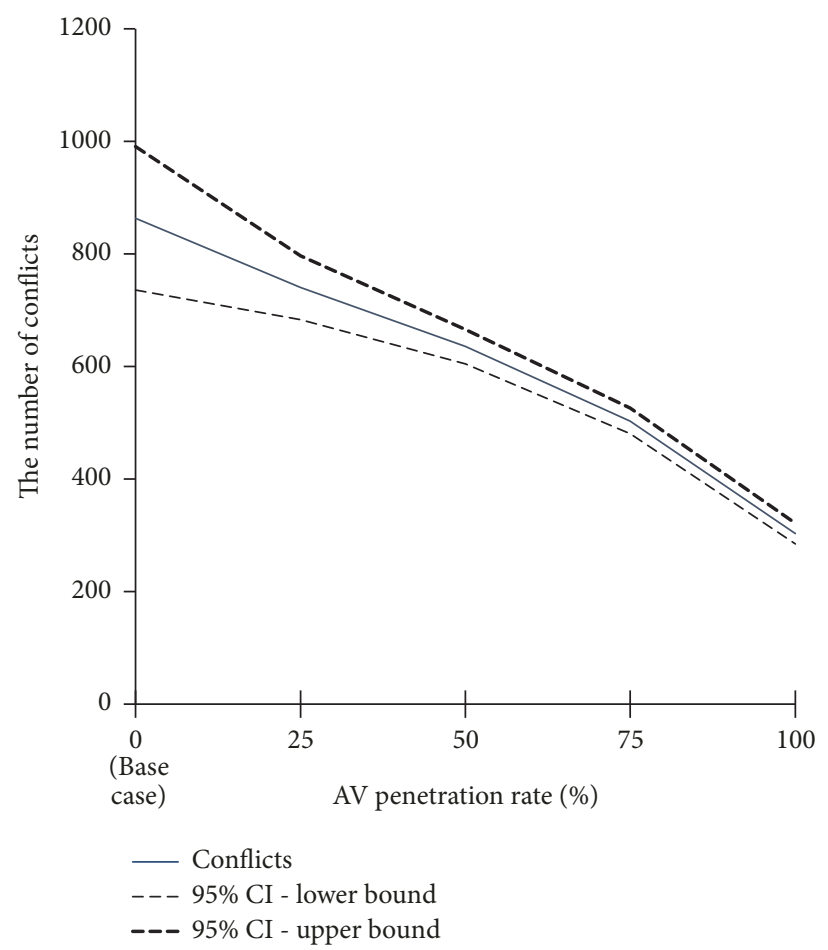

(b) $0.75 \mathrm{~s}$ TTC threshold for AV-AV conflicts

FIGURE 9: Total number of conflicts by AV penetration rate for the signalised intersection (AV-2 parameters).

Overall, results suggest that safety benefits of AVs can be achieved without compromising positive impacts on capacity associated with shorter headways.

3.2. AV-2 Parameters. Results with the AV-2 parameters are presented in Figures 9 and 10 for the signalised intersection and the roundabout, respectively. In general, there are similar patterns in safety benefits of AVs between the two sets of AV parameters. For the signalised intersection, the number of conflicts decreases as the AV penetration rate increases (Figure 9). Particularly with the $100 \%$ AV penetration rate, the number of conflicts continues to decrease significantly. The number of conflicts with the $100 \%$ penetration rate is significantly lower compared to all other penetration rates. Compared to the base case, the number of conflicts with the penetration rate of $50 \%$ or more is significantly lower at $p<$ 0.05 , with improvements ranging from $21 \%$ to $65 \%$. For the roundabout, the number of conflicts is reduced significantly with the AV penetration rate of $75 \%$ and $100 \%$ (Figure 10 ). Particularly with the $100 \%$ AV penetration rate, the number of conflicts is significantly lower at $p<0.05$ with improvements of $32 \%$ to $64 \%$ compared to the base case, depending on the AV-AV TTC threshold.

The benefits obtained from AV-2 parameters are higher compared to those from AV-1 parameters (maximum benefits of $64 \%$ versus $62 \%$ for the roundabout and $65 \%$ versus $52 \%$ for the signalised intersection). It is noted that the AV-2 parameter set has higher standstill and following variation distances whereas the AV-1 parameter set has slightly higher headway, but more aggressive acceleration. Nevertheless, both AV parameter sets suggest safety benefits of AVs with high penetration rates.

Similarly, with the AV-2 set of parameters, at an increasing penetration rate, there are delay reductions for both the roundabout and the signalised intersection cases (Figure 11). The results in both cases show that reductions in delay with increasing penetration rates of $\mathrm{AVs}$ are statistically significant at $p<0.05$. This further advocates the notion that at increasing $\mathrm{AV}$ penetration rates the system will become more efficient as well as safer.

\section{Conclusion}

This paper has investigated the safety impact of autonomous vehicles (AVs) using traffic microsimulation and Surrogate Safety Assessment Model (SSAM). Safety performance of AVs (level 4 automation, fully automated) in signalised intersection and roundabout case studies was explored through the number of potential conflicts based on time to collision (TTC) and postencroachment time (PET). VISSIM was adopted as the traffic microsimulation platform to model behaviours of human-driven vehicles (HVs) and AVs. More assertive behaviours of AVs, such as shorter headways and more aggressive acceleration, were explicitly considered. Results suggest that AVs improve safety significantly with high penetration rates, even when they travel with shorter headways to improve road capacity and reduce delay. For the signalised intersection, $\mathrm{AVs}$ reduce the number of conflicts by $20 \%$ to $65 \%$ with the penetration rates between $50 \%$ and $100 \%$ (statistically significant at $p<0.05$ ). For the roundabout, 


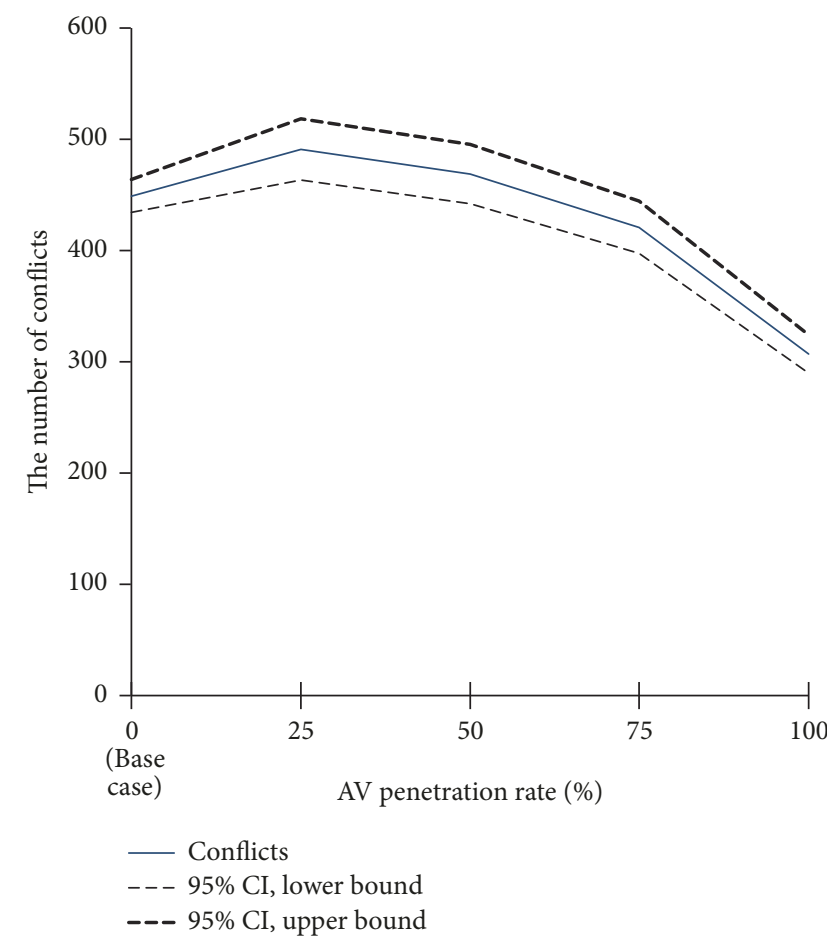

(a) $1 \mathrm{~s}$ TTC threshold for AV-AV conflicts

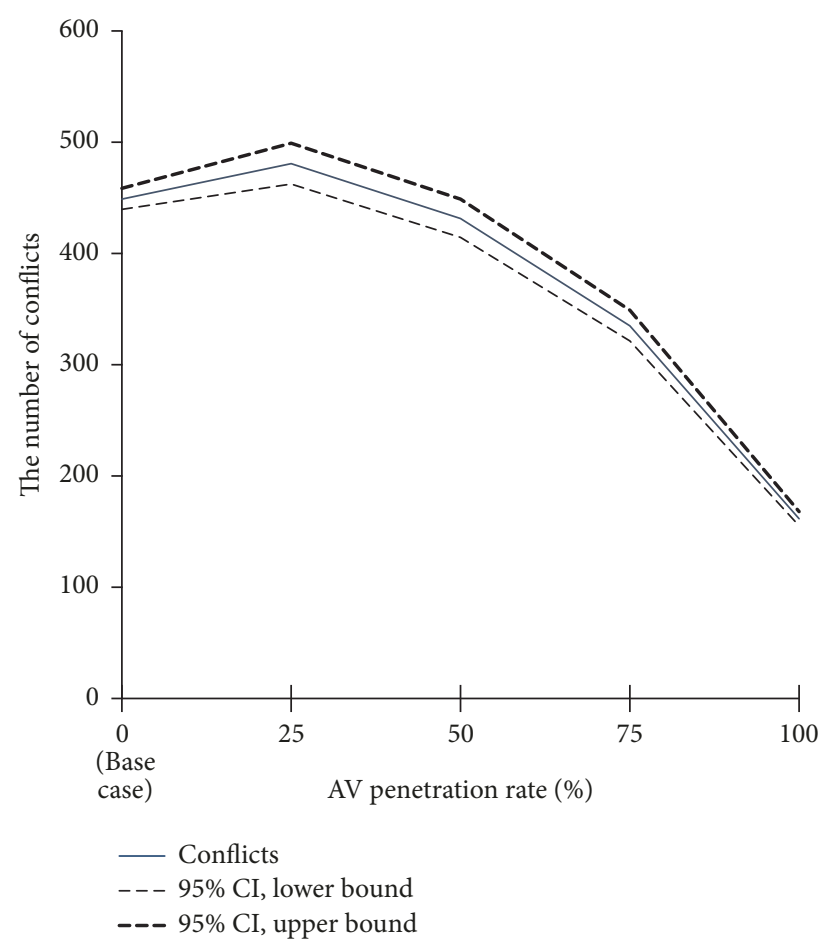

(b) $0.75 \mathrm{~s}$ TTC threshold for AV-AV conflicts

FIgURE 10: Total number of conflicts by AV penetration rate for the roundabout (AV-2 parameters).

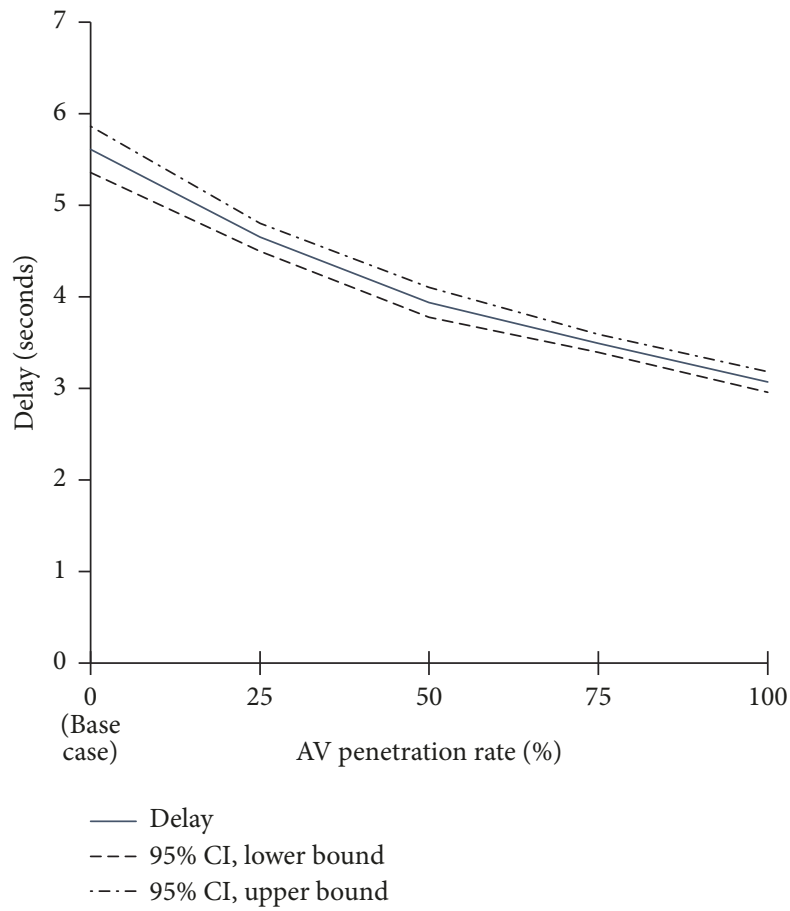

(a) Roundabout delay

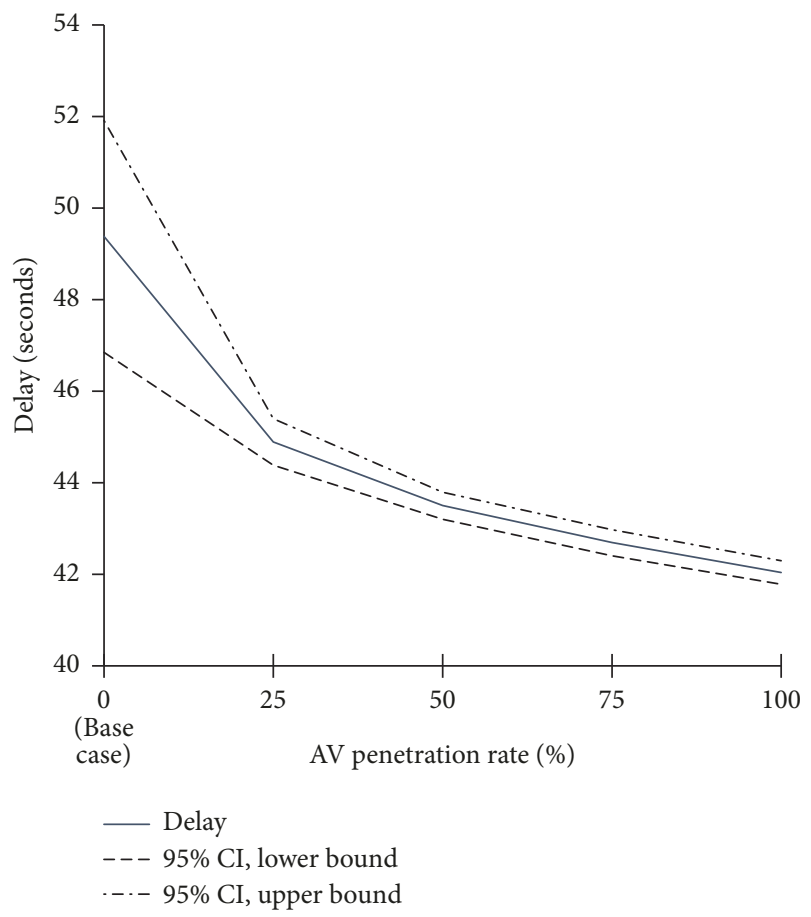

(b) Signalised intersection delay

FIgURE 11: Delay results at an increasing AV penetration rate. 
reductions of between $29 \%$ and $64 \%$, in terms of conflicts, are evident with the $100 \%$ AV penetration rate (statistically significant at $p<0.05$ ). An implication of these findings is that a high AV penetration rate might be required to deliver AVs' anticipated safety benefits.

The simulation-based approach presented in this paper provides an important tool to evaluate safety impacts of AVs, particularly when there has been very limited empirical data on safety performance of AVs. Nevertheless, there are limitations in the proposed approach, which should be addressed in future research. First, the ability to replicate how AVs will act within a real-world road network is limited due to the fact that AV technology is still being developed. Particularly, interactions between human drivers and AVs are largely unknown. Although this study modelled AV behaviours by modifying VISSIM's car-following model in accordance with the recent literature, there is a clear scope to develop a more realistic model for connected AVs. It is also noted that safety benefits of AVs would be higher with connected vehicle technology and control. Future research should also explore the impacts of vehicle-to-vehicle (V2V) safety technology, vehicle platooning, and V2V and vehicle trajectory control. Next, potential conflicts in this analysis were solely based on TTC and PET. The use of other emerging surrogate safety measures, such as Aggregated Crash Index (ACI) [39], should be considered to increase the validity of the approach. It might be necessary to develop new surrogate safety measures for AVs due to their different behaviours. Finally, the study only considered two case studies, a signalised intersection and a roundabout. Therefore, to draw more reliable conclusions about the overall safety impacts of AVs on a large road network, more testing with various network settings under wide-ranging traffic conditions and $\mathrm{AV}$ penetration rates might be required.

\section{Disclosure}

An earlier version of this paper was presented at the Australasian Transport Research Forum (ATRF 2017) in Auckland, New Zealand.

\section{Conflicts of Interest}

The authors declare that there are no conflicts of interest regarding the publication of this paper.

\section{Supplementary Materials}

An animation is provided to illustrate shorter gaps between $\mathrm{AVs}$ in the signalised intersection case study. AVs are yellow and green while HVs are blue in the animation. (Supplementary Materials)

\section{References}

[1] HLDI, Honda Accord collision avoidance features: an update. Bulletin Vol. 31. No. 16, Highway Loss Data Institute, Insurance Institute for Highway Safety, Arlington, Va, USA, 2014.
[2] J. Bierstedt, A. Gooze, C. Gray, J. Peterman, L. Raykin, and J. Walters, "Effects of Next-Generation Vehicles on Travel Demand and Highway Capacity," FP Think, 2014.

[3] DPTI, SA becomes first Australian jurisdiction to allow on-road driverless car trials, Department of Planning, Transport and Infrastructure, 2016.

[4] T. Litman, Autonomous Vehicle Implementation Predictions: Implications for Transport Planning, Victoria Transport Policy Institute, 2015.

[5] P. Bansal and K. M. Kockelman, "Forecasting Americans' longterm adoption of connected and autonomous vehicle technologies," Transportation Research Part A: Policy and Practice, vol. 95, pp. 49-63, 2017.

[6] BITRE, Fatal road crashes in Australia in the 1990s and 2000s: crash types and major factors, Bureau of Infrastructure, Transport and Regional Economics, Canberra, Australia, 2011.

[7] NHTSA, National Motor Vehicle Crash Causation Survey, National Highway Traffic Safety Administration, U.S. Department of Transportation, 2008.

[8] S. E. Shladover, "Cooperative (rather than autonomous) vehicle-highway automation systems," IEEE Intelligent Transportation Systems Magazine, vol. 1, no. 1, pp. 10-19, 2009.

[9] R. Hoogendoorn, B. Van Arem, and S. Hoogendoorn, "Automated driving, traffic flow efficiency, and human factors," Transportation Research Record, vol. 2422, pp. 113-120, 2014.

[10] M. A. S. Kamal, J.-I. Imura, T. Hayakawa, A. Ohata, and K. Aihara, "A vehicle-intersection coordination scheme for smooth flows of traffic without using traffic lights," IEEE Transactions on Intelligent Transportation Systems, vol. 16, no. 3, pp. 1136-1147, 2015.

[11] L. T. Truong, C. de Gruyter, G. Currie, and A. Delbosc, "Estimating the trip generation impacts of autonomous vehicles on car travel in Victoria, Australia," Transportation, vol. 44, no. 6, pp. 1279-1292, 2017.

[12] D. Milakis, B. Van Arem, and B. Vanwee, "Policy and society related implications of automated driving: A review of literature and directions for future research," Journal of Intelligent Transportation Systems: Technology, Planning, and Operations, vol. 21, no. 4, pp. 324-348, 2017.

[13] S. Childress, B. Nichols, B. Charlton, and S. Coe, "Using an activity-based model to explore possible impacts of automated vehicles," Transportation Research Record: Journal of the Transportation Research Board, vol. 2493, pp. 99-106, 2015.

[14] D. J. Fagnant and K. Kockelman, "Preparing a nation for autonomous vehicles: Opportunities, barriers and policy recommendations," Transportation Research Part A: Policy and Practice, vol. 77, pp. 167-181, 2015.

[15] P. Koopman and M. Wagner, "Autonomous vehicle safety: an interdisciplinary challenge," IEEE Intelligent Transportation Systems Magazine, vol. 9, no. 1, pp. 90-96, 2017.

[16] V. V. Dixit, S. Chand, and D. J. Nair, "Autonomous vehicles: disengagements, accidents and reaction times," PLoS ONE, vol. 11, no. 12, Article ID 0168054, 2016.

[17] P. Rau, M. Yanagisawa, and W. G. Najm, "Target crash population of automated vehicles," in Proceedings of the In 24th International Technical Conference on the Enhanced Safety of Vehicles (ESV), Gothenburg, Sweden, 2015.

[18] B. Schoettle and M. Sivak, A Preliminary Analysis of RealWorld Crashes Involving Self-Driving Vehicles, The University of Michigan, Ann Arbor, Mich, USA, 2015. 
[19] P. Bhavsar, P. Das, M. Paugh, K. Dey, and M. Chowdhury, "Risk Analysis of Autonomous Vehicles in Mixed Traffic Streams," Transportation Research Record, vol. 2625, pp. 51-61, 2017.

[20] F. M. Favarò, N. Nader, S. O. Eurich, M. Tripp, and N. Varadaraju, "Examining accident reports involving autonomous vehicles in California," PLoS ONE, vol. 12, no. 9, p. e0184952, 2017.

[21] D. Tian, J. Zhou, Y. Wang, Z. Sheng, H. Xia, and Z. Yi, “Modeling chain collisions in vehicular networks with variable penetration rates," Transportation Research Part C: Emerging Technologies, vol. 69, pp. 36-59, 2016.

[22] Y. Wei, C. Avc1, J. Liu et al., "Dynamic programming-based multi-vehicle longitudinal trajectory optimization with simplified car following models," Transportation Research Part B: Methodological, vol. 106, pp. 102-129, 2017.

[23] M. Zhou, X. Qu, and S. Jin, "On the impact of cooperative autonomous vehicles in improving freeway merging: a modified intelligent driver model-based approach," IEEE Transactions on Intelligent Transportation Systems, vol. 18, no. 6, pp. 1422-1428, 2017.

[24] K. Kockelman, P. Avery, P. Bansal et al., "Implications of Connected and Automated Vehicles on the Safety and Operations of Roadway Networks," Final Report, Texas Department of Transportation, Austin, Tex, USA, 2016.

[25] PTV, "VISSIM 9 - User Manual," PTV AG, Karlsruhe, Germany, 2016.

[26] D. Gettman, L. Pu, T. Sayed, and S. Shelby, "Surrogate Safety Assessment Model and Validation," Final Report, Federal Highway Administration, McLean, Va, USA, 2008.

[27] NHTSA, U.S. Department of Transportation Releases Policy on Automated Vehicle Development, National Highway Traffic Safety Administration, U.S. Department of Transportation, 2013.

[28] Atkins, "Research on the Impacts of Connected and Autonomous Vehicles (CAVs) on Traffic Flow," Stage 2: Traffic Modelling and Analysis Technical Report, Department for Transport, 2016.

[29] PTV, Autonomous vehicle lateral space - example description, PTV AG, Karlsruhe, Germany, 2017.

[30] D. Gettman and L. Head, "Surrogate Safety Measures From Traffic Simulation Models," Final Report, Federal Highway Administration, McLean, Va, USA, 2003.

[31] K. Goh, G. Currie, M. Sarvi, and D. Logan, "Experimental microsimulation modeling of road safety impacts of bus priority," Transportation Research Record, no. 2402, pp. 9-18, 2014.

[32] W. Young, A. Sobhani, M. G. Lenné, and M. Sarvi, "Simulation of safety: A review of the state of the art in road safety simulation modelling," Accident Analysis \& Prevention, vol. 66, pp. 89-103, 2014.

[33] M. Essa and T. Sayed, "A comparison between PARAMICS and VISSIM in estimating automated field-measured traffic conflicts at signalized intersections," Journal of Advanced Transportation, vol. 50, no. 5, pp. 897-917, 2016.

[34] N. Saunier, "Surrogate Safety Analysis," Polytechnique Montréal, 2010.

[35] P. Chen, W. Zeng, G. Yu, and Y. Wang, "Surrogate safety analysis of pedestrian-vehicle conflict at intersections using unmanned aerial vehicle videos," Journal of Advanced Transportation, vol. 2017, Article ID 5202150, 12 pages, 2017.

[36] L. T. Truong, M. Sarvi, G. Currie, and T. M. Garoni, "How many simulation runs are required to achieve statistically confident results: a case study of simulation-based surrogate safety measures," in Proceedings of the 18th IEEE International Conference on Intelligent Transportation Systems (ITSC '15), pp. 274-278, Las Palmas de Gran Canaria, Spain, September 2015.

[37] A. Dijkstra, P. Marchesini, F. Bijleveld, V. Kars, H. Drolenga, and M. V. Maarseveen, "Do calculated conflicts in microsimulation model predict number of crashes?" Transportation Research Record, no. 2147, pp. 105-112, 2010.

[38] L. T. Truong, M. Sarvi, G. Currie, and T. M. Garoni, "Required traffic micro-simulation runs for reliable multivariate performance estimates," Journal of Advanced Transportation, vol. 50, no. 3, pp. 296-314, 2016.

[39] Y. Kuang, X. Qu, and S. Wang, "A tree-structured crash surrogate measure for freeways," Accident Analysis \& Prevention, vol. 77, pp. 137-148, 2015. 


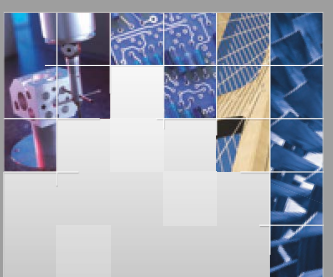

\section{Enfincering}
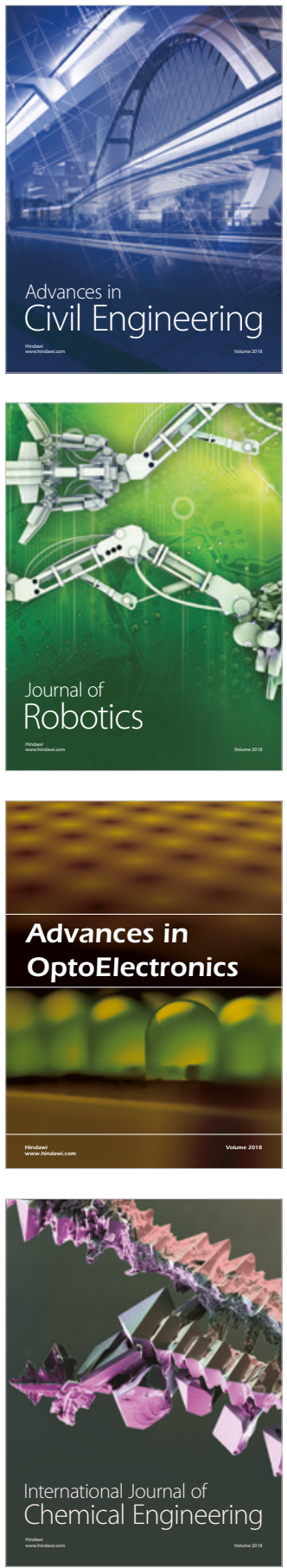

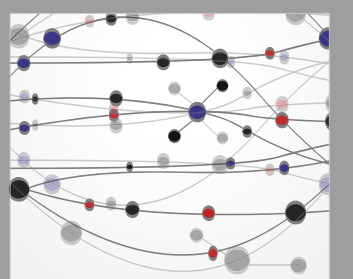

\section{Rotating \\ Machinery}

The Scientific World Journal

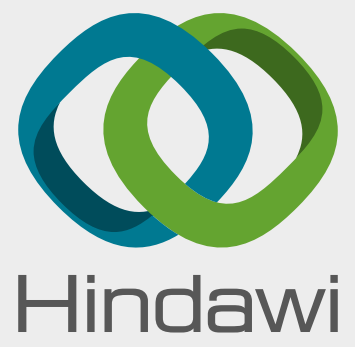

Submit your manuscripts at

www.hindawi.com
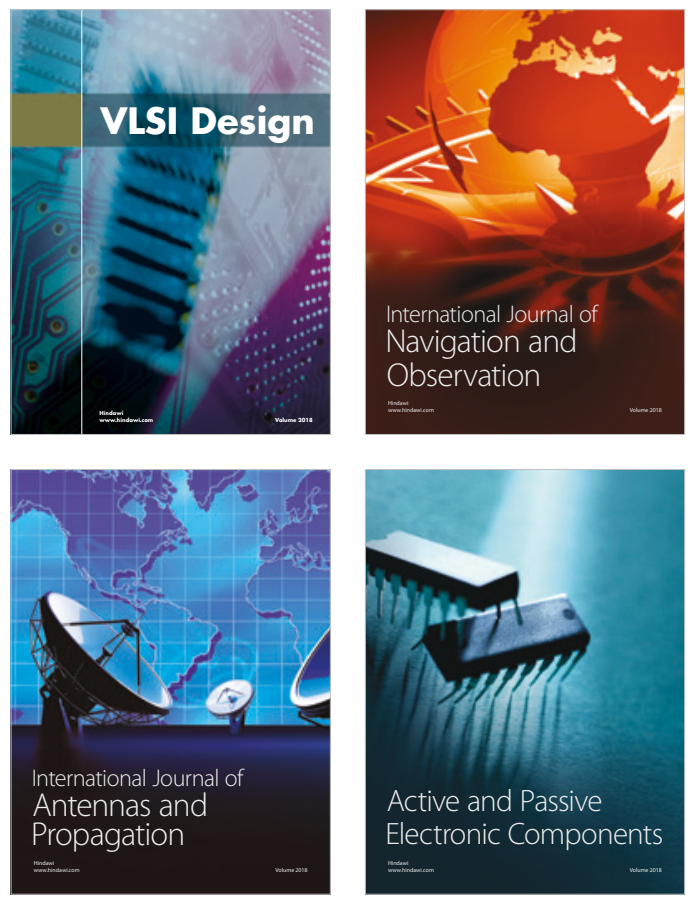
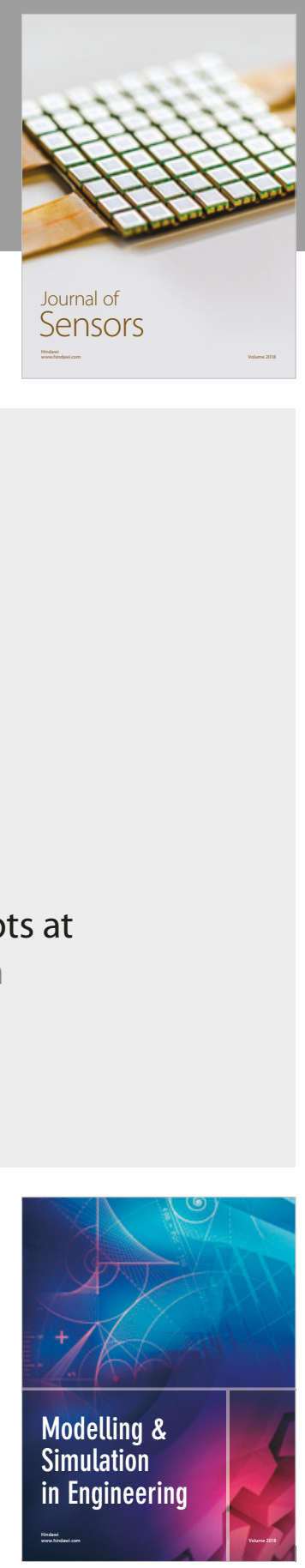

\section{Advances \\ Multimedia}
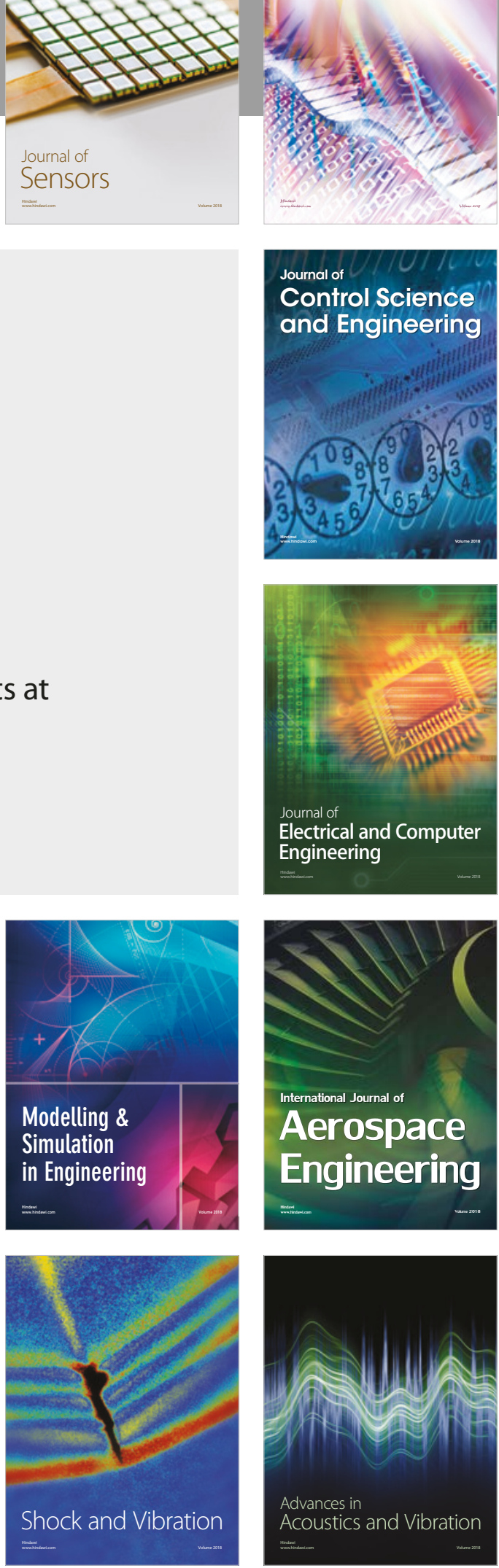